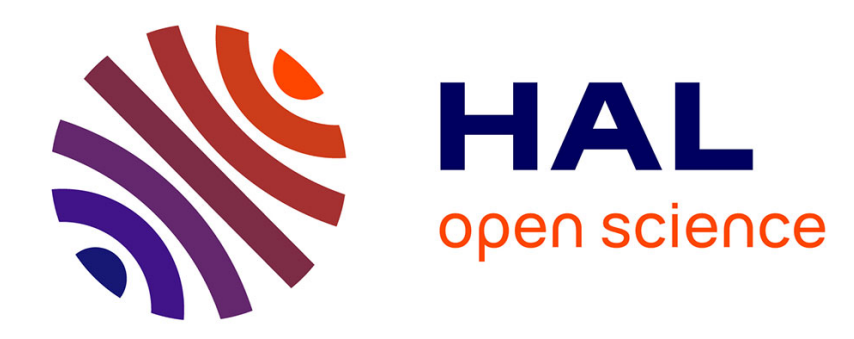

\title{
Deformable Random Dot Markers
}

H. Uchiyama, E. Marchand

\section{- To cite this version:}

H. Uchiyama, E. Marchand. Deformable Random Dot Markers. IEEE Int. Symp. on Mixed and Augmented Reality, ISMAR'11, 2011, Basel, Switzeland, Switzerland. pp.237-238. hal-00639703

\section{HAL Id: hal-00639703 https://hal.inria.fr/hal-00639703}

Submitted on 9 Nov 2011

HAL is a multi-disciplinary open access archive for the deposit and dissemination of scientific research documents, whether they are published or not. The documents may come from teaching and research institutions in France or abroad, or from public or private research centers.
L'archive ouverte pluridisciplinaire HAL, est destinée au dépôt et à la diffusion de documents scientifiques de niveau recherche, publiés ou non, émanant des établissements d'enseignement et de recherche français ou étrangers, des laboratoires publics ou privés. 


\section{Deformable Random Dot Markers}

\author{
Hideaki Uchiyama* \\ INRIA Rennes Bretagne-Atlantique
}

\author{
Eric Marchand ${ }^{\dagger}$ \\ Université de Rennes 1, IRISA, \\ INRIA Rennes Bretagne-Atlantique
}
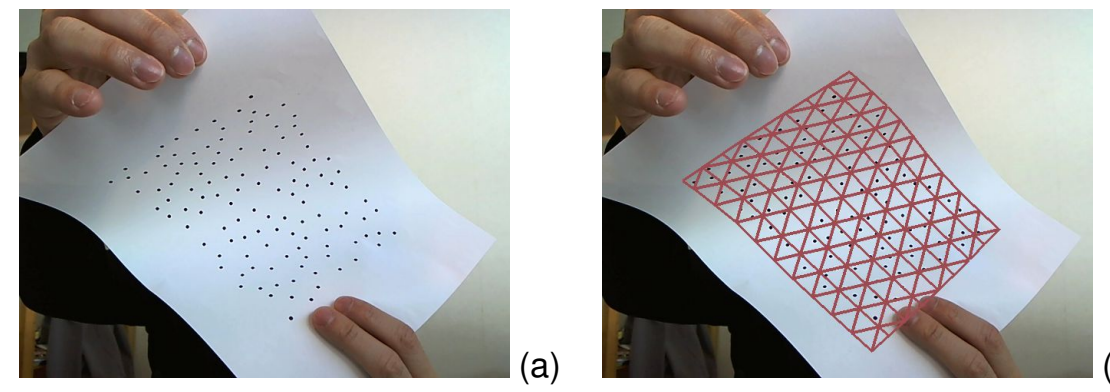

(b)

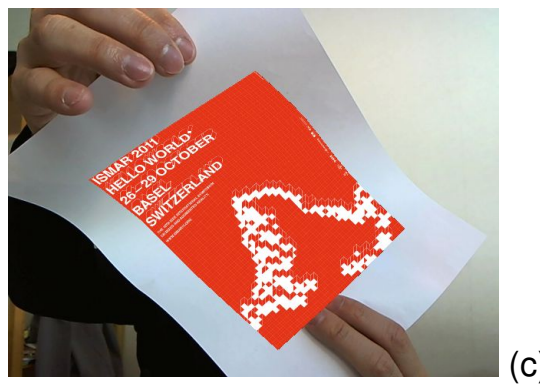

Figure 1: Nonrigidly deformable random dot markers. (a) For an input image, (b) the deformed surface of a marker is recovered from keypoint correspondences using random dots, (c) texture is overlaid on the deformed surface.

\section{Abstract}

We extend planar fiducial markers using random dots [8] to nonrigidly deformable markers. Because the recognition and tracking of random dot markers are based on keypoint matching, we can estimate the deformation of the markers with nonrigid surface detection from keypoint correspondences. First, the initial pose of the markers is computed from a homography with RANSAC as a planar detection. Second, deformations are estimated from the minimization of a cost function for deformable surface fitting. We show augmentation results of $2 \mathrm{D}$ surface deformation recovery with several markers.

Index Terms: H.5.1 [INFORMATION INTERFACES AND PRESENTATION (e.g., HCI)]: Multimedia Information SystemsArtificial, augmented, and virtual realities; I.4.8 [IMAGE PROCESSING AND COMPUTER VISION]: Scene AnalysisTracking

\section{INTRODUCTION}

Fiducial markers have been utilized in a wide field of research. For example, the initialization of a camera pose in visual SLAM can be done with a marker to estimate a known scale. Studies on mixed/augmented reality have also been proposed such that a camera pose with respect to real world can be estimated to overlay virtual objects with geometrical consistency. Thanks to their simplicity of use, the stability of recognition and tracking process, up to now fiducial markers have been a useful tool and will continue to be utilized in the future as well.

The shape of planar fiducial markers was generally square with a black frame such as ARToolKit [3] and ARTag [1]. As another type of fiducial marker, random dot markers were proposed [8]. Compared with traditional square markers, the flexibility of design and the occlusion robustness were improved. However, random dot

*e-mail: Hideaki.Uchiyama@inria.fr

†e-mail: Eric.Marchand@irisa.fr markers should still be planar as well as traditional markers. In order to enhance the functionality of fiducial markers, we propose an approach for recognizing and tracking nonrigidly deformable random dot markers.

\section{Related Works}

The world first AR system with fiducial markers was developed by Rekimoto [7]. The markers had a black and white coded square pattern inside a black frame and were recognized by decoding the pattern. The shape of binary pattern can be replaced with hexagon [9], circle [4] and frame [10]. Generally, such square markers are not able to be deformed because the pose of the markers is computed from the position of the four corners using homography.

Random dot markers have been developed to relax the constraints for the design and usability of fiducial markers [8]. Each dot is first extracted with binarization and utilized as a keypoint. The recognition and tracking of the markers are based on keypoint matching using local geometrical relationship of keypoints with LLAH (Locally Likely Arrangement Hashing) [5]. It was experimentally confirmed that 40 random dots per marker were distinguishable enough for recognizing 1000 markers. Because the augmentation of a deformed surface can be useful for several AR systems, we extend this work to deformable random dot markers.

\section{Detecting and Tracking Deformation}

In this section, we describe the procedure of recognizing the markers and tracking their deformation.

\subsection{Nonrigid Surface Recovery}

Zhu and Lyu nicely formulated the problem of nonrigid surface recovery from keypoint correspondences such that the shape of 2D nonrigid surface could be computed by solving two linear equations with the finite number of iteration [11]. The complexity of its computation is equivalent to one Newton optimization step. Because of low computational cost and the simplicity of the implementation, we incorporate this method into our approach. 


\subsection{Setup}

As in [8], we first generate random dots without any overlay. In a keypoint database, the geometrical descriptors of each dot are stored. In addition, we decide the number of triangles and their size on a mesh as illustrated in Figure 2. The number of triangles is closely correlated with computational cost for recovering the shape.

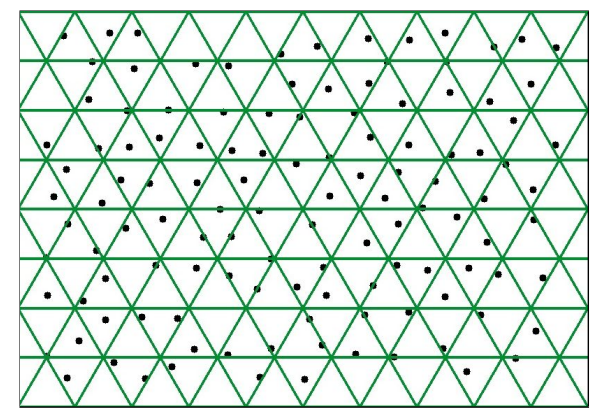

Figure 2: Mesh on a marker. A marker is represented by triangles with hexagonally connected vertices. The more the number of triangles increases, the more computational power is required.

\subsection{Recognizing and Tracking Multiple Markers}

For marker recognition, a marker is initially set as a plane and recognized by computing RANSAC based homography as in [8]. Once a marker is recognized, the detected plane is utilized as the reference plane for the Zhu's method [11].

During tracking the deformation of a marker, the keypoints in a current image is matched with those in the previous image using LLAH [5]. From these correspondences, we estimate the surface shape only with one iteration in the optimization of the Zhu's method because the deformation between consecutive images is small. This continuous estimation of the surface shape works as the tracking of marker deformation. As illustrated in Figure 3 (a) and (b), the marker shape can nonrigidly be deformed. We can consider that tracking fails when the number of inliers is lower than a threshold because the Zhu's method returns the inliers from the keypoint correspondences.

The overall procedure for recognizing and tracking multiple markers is as follows. First, all keypoints extracted in an image are matched with a keypoint database as marker recognition. To recognize multiple markers simultaneously, RANSAC based homography is computed for several candidates of markers that have enough correspondences as in [8]. Once markers are recognized, the keypoints in those markers are tracked by matching with the keypoints in the next frame using LLAH. For the remaining of the keypoints, that are not tracked in the image, marker recognition is again performed to find new markers. As illustrated in Figure 3 (c), multiple markers can simultaneously be recognized and tracked.

Note that the computational cost for recognizing one marker is not much affected by the number of markers registered in a database because LLAH utilizes a hash scheme for finding a keypoint correspondence from the database [5]. The cost is mainly affected by the number of keypoints extracted in an image. This means that the cost increases when the number of markers captured in an image increases. Tracking the deformation of one marker can run over 30 frames per second on an Intel Core i7 M620 (2.67GHz).

\section{Conclusion}

We proposed an approach for recognizing and tracking multiple deformable fiducial markers using random dots. Because our marker
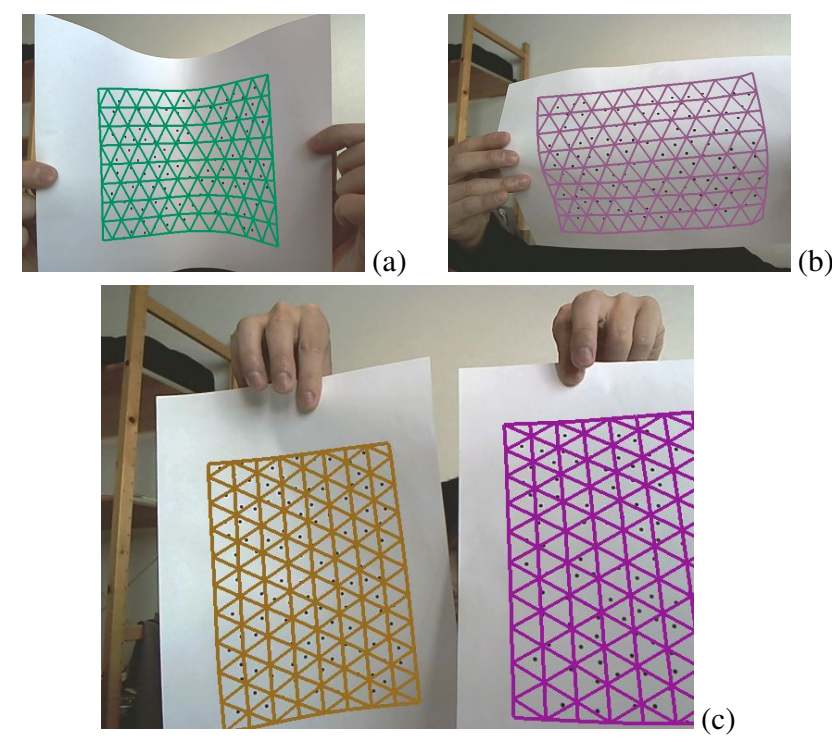

(b)

Figure 3: Augmentation results. A marker can nonrigidly be deformed as (a) and (b). (c) Multiple markers can simultaneously be recognized and tracked.

system is based on keypoint matching, we utilized nonrigid surface recovery from keypoint correspondences as considered in [11]. Other methods for nonrigid surface recovery such as [6] and [2] can also be applicable to our approach.

One of the future works is the retexturing of the surface considering lighting conditions as in [11]. The quality of retexturing random dot markers will be good because random dots can easily be removed by covering the dots with the color of the background. Also, we will develop some applications such that a virtual cloth can be overlaid on a white shirt with random black dot as an AR clothing system.

\section{REFERENCES}

[1] M. Fiala. ARTag, a fiducial marker system using digital techniques. In CVPR, pages 590-596, 2005.

[2] V. Gay-Bellile, A. Bartoli, and P. Sayd. Direct estimation of nonrigid registrations with image-based self-occlusion reasoning. TPAMI, 32(1):87-104, 2010.

[3] H. Kato and M. Billinghurst. Marker tracking and HMD calibration for a video-based augmented reality conferencing system. In IWAR, pages 85-94, 1999.

[4] L. Naimark and E. Foxlin. Circular data matrix fiducial system and robust image processing for a wearable vision-inertial self-tracker. In ISMAR, pages 27-36, 2002.

[5] T. Nakai, K. Kise, and M. Iwamura. Use of affine invariants in locally likely arrangement hashing for camera-based document image retrieval. In $D A S$, pages 541-552, 2006.

[6] J. Pilet, V. Lepetit, and P. Fua. Fast non-rigid surface detection, registration and realistic augmentation. IJCV, 76(2):109-122, 2008.

[7] J. Rekimoto. Matrix: a realtime object identification and registration method for augmented reality. In APCHI, pages 63-68, 1998.

[8] H. Uchiyama and H. Saito. Random dot markers. In IEEE VR, pages 35-38, 2011.

[9] S. Uchiyama, K. Takemoto, K. Satoh, H. Yamamoto, and H. Tamura. MR platform: a basic body on which mixed reality applications are built. In ISMAR, pages 246-253, 2002.

[10] D. Wagner, T. Langlotz, and D. Schmalstieg. Robust and unobtrusive marker tracking on mobile phones. In ISMAR, pages 121-124, 2008.

[11] J. Zhu and M. R. Lyu. Progressive finite newton approach to real-time nonrigid surface detection. In CVPR, 2007. 